\section{Assessment of urinalysis reflex to culture criteria: Impact on antimicrobial usage}

Mohammad Ourani ${ }^{1}$, Nathan S Honda?, William MacDonald², Jill Roberts ${ }^{3}$

1 Department of Pathology and Laboratory Services, PIH health in Whittier, CA, USA.

2 Department of Pathology and Laboratory Services, PIH health in Downey, CA, USA.

3 College of Public Health, University of South Florida, FL, USA.

\section{Contact information:}

\section{Mohammad Ourani.}

Culture (UARC) in a 400-bed acute care hospital. A total of 4016 urine samples were collected and examined between February and April 2020. The UA results were then subjected to the laboratory UA reflex criteria for reflecting UA to culture. Multivariable logistic regression was utilized in evaluating the effectiveness of the criteria's parameters to predict positive urine cultures.

Results: The total number of the positive UA reflex samples was 1539, which accounted for $38.3 \%$ of all the UA samples. Moreover, those positive UA samples were reflexed to urine cultures. Among the urine samples that were cultured, $45.1 \%(n=694)$ were negative urine cultures while $54.9 \%(n=845)$ were positive urine cultures. The UA reflex criteria was associated with positive predictive values for positive urine cultures between $26.30 \%$ and $92.96 \%$.

Conclusions: The current Laboratory UA reflex criteria is not highly effective in predicting positive urine culture, thus potentially leading to the inappropriate use of antibiotics.
Address: College of Public Health, University of South Florida, FL, USA.

Đouranim@usf.edu

Keywords

Urinary Tract Infection, Urinalysis, Urine Culture, Antibiotics Usage, USA.

\title{
Introduction
}

Antimicrobial resistance $(A R)$ is a serious threat to the treatment of infectious diseases, which might result in human side effects, loss of life, and huge economic impacts. Several efforts are taking place 
to combat $A R$ and halt the spread of resistance. Laboratories play a vital role in the prevention of the spread of $A R$ [1]. The microbiology laboratory can rapidly detect $A R$ pathogens and inform treating physicians and infection control practitioners to help prevent the spread and protect other patients (CDC 2018). Most importantly, optimal diagnosis of various infections is essential as it ensures the appropriate antibiotic treatment and prevents unnecessary use of antibiotics. For instance, urinary tract infections (UTIS) are one of the leading conditions that require antibiotics intervention, hence contributing highly to antibiotic misuse [2]. Patients presented with the possibility of having urinary tract infection (UTI) are often screened by urinalysis (UA) to determine whether administration of antibiotics is warranted and then whether a urine culture is needed. Unfortunately, improper urinalysis reflex criteria can result in suboptimal laboratory practice and can incorrectly influence healthcare practitioners to inappropriately prescribe antibiotics [3-4].

Urinalysis is a rapid diagnostic tool utilized by healthcare providers to clarify uncertainty in the diagnosis of UTI. However, laboratories must adhere to evidence-based practices and high predictive UA diagnostic criteria to ensure providing accurate patient diagnosis and appropriate antibiotics treatment. Most laboratory practices rely on historical UA reflex criteria that have not been updated for many years, thus, they are often associated with a high rate of false positives UAs $[1,5]$. This was evident in the baseline assessment during this study, where we reported high false positives due to the application of outdated UA reflex criteria applied in our laboratory. Suboptimal or improper laboratory testing is a major challenge and a serious quality problem, significantly affecting patient's outcome. In particular, the reflex criteria rely on a single UA reflex parameter to predict positive urine cultures which were associated with a low positive predictive value. Consequently, they influence the providers towards the suboptimal administration of antibio- tics. According to the Centers for Disease Control (CDC), over-testing and over-treatment are linked to excessive usage of antibiotics and result in unnecessary healthcare costs of more than $\$ 1.1$ billion annually [6-8].

\section{Methods}

A prospective study using laboratory data was conducted on inpatient urine samples with orders placed for Urinalysis Reflex Culture (UARC) in a 400-bed acute care hospital. A total of 4016 urine samples (2426 females, 1590 males) between age of 2 and 75 years old with a median age of 43 years. Samples were collected and examined between February and March 2020. The purpose of this study is to assess the predictive value of the UA reflex criteria's parameters and to evaluate the criteria's impact on usage of antibiotics. The study was evaluated by the hospital regulatory department, and it was determined to have the legal or ethical status of "quality improvement activity" rather than "human subject research." Urine samples were collected from patients who presented to the hospital for routine workup or possible UTIs but not necessarily with any signs or symptoms. As per Clinical and Laboratory Standards Institutes (CLSI) urinalysis guidelines (CLSI, 2009), nurses or laboratory staff provided patients with a clean-catch urine collection instruction to minimize contamination caused by bacteria on the skin. Following the instructions provided, a minimum of $5 \mathrm{ml}$ urine clean-catch sample was collected from patients in a sterile urine cup which was submitted to nursing or laboratory staff in a timely manner. Samples were transported to the laboratory to be tested within two hours of collection if were unrefrigerated or within 24 hours if kept refrigerated. Urine samples were tested for urinalysis on the AUTION HYBRID AU-4050 (Arkray, Minneapolis, MN), Microscopic examination was performed if the urine was positive for any of the followings; red blood cells (2- 
Vol. 10 No. 3:4

doi: $10.3823 / 851$

5), nitrite(+), protein (+) or leukocyte esterase (+). Urinalysis is defined as positive if it contains any of these parameters: nitrites+, leukocyte esterase+, bacteria present, or (0-5) white blood cells present per high-power field (hpf). UA-reflexed data statistics and antibiotic usage were recorded during the baseline period.

Statistical data were extracted through the laboratory information system (TD Synergy) a product of Tech Data, Inc,USA, and the pharmacy electronic inventory system (SRX-Allscripts) a product of All scripts Health care Solutions, Inc. Multivariable logistic regression was utilized to evaluate the predictive value of UA criteria's parameters and to identify the highest predictive reflex indicators with clinical significance that resulted in true positive urine cultures. The statistical analysis of the results was analyzed and interpreted using IBM SPSS Statistics software (V25.0).

\section{Results}

A total of 4016 patients participated with samples in the study, among which $60.4 \%(n=2426)$ were females and $39.6 \%(n=1590)$ were males. The total number of the negative UA reflex samples was 2477 which accounted for $61.7 \%$ of all the UA reflex samples. In addition, $38.3 \%$ of all the UA positive samples ( $n=1539$ ) were reflexed to urine culture. Among the urine samples that were cultured, $45.1 \%(n=694)$ were negative urine cultures, while $54.9 \%(n=845)$ were positive urine cultures. See Table 1.

\section{Evaluation of bacteria presence as a single parameter to predict positive urine culture}

Bacteriuria is the presence of bacteria in urine and when accompanied by symptoms will most likely be an indication of a urinary tract infection. Gram negative rod bacteria such Escherichia coli is the most common cause of UTIs. Bacteria presence in
Table 1. Summary of the study descriptive statistics.

\begin{tabular}{|l|c|c|}
\hline \multicolumn{1}{|c|}{ Characters } & Frequency & Percent \\
\hline Gender & & \\
\hline Female & 2426 & 60.4 \\
\hline Male & 1590 & 39.6 \\
\hline Total no. & 4016 & 100 \\
\hline UA Reflex Samples & & \\
\hline Negative & 2477 & 61.7 \\
\hline Positive & 1539 & 38.3 \\
\hline Total no. & 4016 & 100 \\
\hline UC & & \\
\hline Negative & 694 & 45.1 \\
\hline Positive & 845 & 54.9 \\
\hline Total no. & 1539 & 100 \\
\hline
\end{tabular}

urine as a single UA parameter with a cut-off value of (0-1) showed $30.1 \%$ PPV for positive urine culture; $(214 /(214+498)=30.1 \%$, while bacteria presence with a cut-off value of $>1$ showed $76.3 \%$ PPV for positive urine culture; $(631 /(631+196)=76.3 \%$ as described in Table 2.

Table 2. Bacteria prediction for positive urine cultures.

\begin{tabular}{|l|c|c|c|c|}
\hline \multirow{2}{*}{ Urine cultures } & \multicolumn{4}{|c|}{ Bacteria (hpf) } \\
\cline { 2 - 5 } & \multicolumn{3}{|c|}{$\mathbf{0 - 1}$} & \multicolumn{2}{c|}{$>1$} \\
\hline Count & $\%$ & Count & $\%$ \\
\hline Negative & 498 & 69.9 & 196 & 23.7 \\
\hline Positive & 214 & 30.1 & 631 & 76.3 \\
\hline Total no. & 712 & 100 & 827 & 100 \\
\hline
\end{tabular}

\section{Evaluation of nitrite as a single UA} parameter to predict positive urine culture Normal urine contains a chemical called nitrate and the presence of certain bacteria; mostly Gramnegative organisms can convert nitrate into nitrite. Therefore, the presence of nitrite gives us indication of the presence of certain bacteria in the urine which indicate the possibility of UTI. In the present study, nitrite negative UAs that resulted 
in positive urine culture showed $41.0 \%$ PPV; (462/ $(665+462)=41.0 \%$, while positive nitrite UAs with positive urine culture showed $92.96 \%$ PPV; (383) $(383+29)=92.96 \%$ as described in Table 3.

Table 3. Nitrite prediction for positive urine cultures.

\begin{tabular}{|l|c|c|c|c|}
\hline \multirow{2}{*}{ Urine cultures } & \multicolumn{4}{|c|}{ Nitrite } \\
\cline { 2 - 5 } & \multicolumn{3}{|c|}{ Negative } & \multicolumn{2}{c|}{ Positive } \\
\cline { 2 - 5 } & Count & $\%$ & Count & $\%$ \\
\hline Negative & 665 & 59 & 29 & 7.04 \\
\hline Positive & 462 & 41 & 383 & 92.96 \\
\hline Total no. & 1127 & 100 & 412 & 100.0 \\
\hline
\end{tabular}

\section{Evaluation of esterase as a single UA} parameter to predict positive urine culture The presence of esterase in the urine indicates the presence of WBCs, which indicate possible UTI. In the present study, trace or few esterase presence in the UA had $33.7 \%$ PPV for positive urine culture; $(299 /(299+588)=33.7 \%$, while moderate or large esterase had $83.7 \%$ PPV for positive urine culture; $(546 /(546+106)=83.7 \%$ as described in Table 4.

Table 4. Esterase prediction for positive urine cultures.

\begin{tabular}{|l|c|c|c|c|}
\hline \multirow{2}{*}{ Urine cultures } & \multicolumn{4}{|c|}{ Esterase } \\
\cline { 2 - 5 } & \multicolumn{2}{|c|}{ Trace or Few } & Moderate or Large \\
\cline { 2 - 5 } & Count & $\%$ & Count & $\%$ \\
\hline Negative & 588 & 66.3 & 106 & 16.3 \\
\hline Positive & 299 & 33.7 & 546 & 83.7 \\
\hline Total no. & 887 & 100 & 652 & 100 \\
\hline
\end{tabular}

\section{Evaluation of the WBCs as a single UA} parameter to predict positive urine culture The presence of WBCs in the urine is indication of a possibility of UTI. In the present study, UA samples with WBCs (0-5) showed $26.30 \%$ PPV for positive urine cultures; $(96 /(96+269)=26.30 \%$, while UA samples with WBCs $>5$ showed $85.09 \%$ PPV for positive urine cultures; $(999 /(999+175)=85.09 \%$ as described in Table 5.

Table 5. WBCs prediction for positive urine cultures.

\begin{tabular}{|l|c|c|c|c|}
\hline \multirow{2}{*}{ Urine cultures } & \multicolumn{4}{|c|}{ WBCs } \\
\cline { 2 - 5 } & \multicolumn{2}{|c|}{$\mathbf{0 - 5}$} & \multicolumn{2}{|c|}{$>\mathbf{5}$} \\
\hline Negative & Count & $\%$ & Count & $\%$ \\
\hline Positive & 269 & 73.70 & 175 & 14.91 \\
\hline Total no. & 96 & 26.30 & 999 & 85.09 \\
\hline
\end{tabular}

\section{Evaluation of the UA reflex criteria's impact on antibiotics usage}

A total of 1539 urine samples that produced positive UA were reflexed to urine culture. Results demonstrated that only $54.9 \%(n=845)$ of the reflexed urine samples showed positive urine culture, which indicated that reliance on the current UA reflex criteria had led to appropriate treatment with antibiotics to $54.9 \%$ of those treated patients. The obtained findings also demonstrated that 694 urine samples with positive UAs yielded negative urine cultures. Consequently, the reliance on the current UA reflex criteria resulted in the inappropriate treatment with antibiotics to $45.1 \%$ of those treated patients. In conclusion, current UA reflex criteria is not optimal and significantly lead to inappropriate use of antibiotics. See Figure 1.

Figure 1: Effectiveness of the current UA reflex criteria on antibiotics usage.

Positive Urinalysis w/antibiotics initiated $n=1539$

\section{Urine Culture Performed}

Negative Urine
Culture
$\mathrm{N}=694$
$45.1 \%$

$\mathbf{4 5 . 1 \%}$ were inappropriately treated with antibiotics

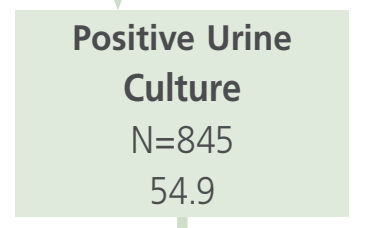

$\mathbf{5 4 . 9 \%}$ were appropriately treated with antibiotics 


\section{Discussion}

Urinalysis is a rapid and relatively simple tool in providing important clinical information for the early diagnosis and treatment of urinary tract infections [9-10]. UTIs are one of the leading conditions that require antibiotics intervention, therefore contributing highly to antibiotic misuse [3, 4, 11-12]. Optimal diagnosis of infections is very vital to ensure appropriate antibiotic treatment and patient recovery [13-14]. On the contrary, suboptimal laboratory test results can generate misleading diagnoses and influence the treating physicians to unnecessarily prescribe antibiotics, consequently leading to the development of antimicrobial resistance $[6,15]$.

Most laboratory practices depend on standard UA reflex criteria that have not been updated for many years; thus, they are often associated with high numbers of false positives $[7,16]$. For example, using cut-off values of $<1$ for bacteria, or any positive nitrite test, or presence of few or less esterase and $<5$ WBCs was associated with low positive predictive values (between $11.4 \%$ and $45.3 \%$ ). The observed low predictive values are associated with the laboratory procedures that are based on reflexing UA for culture if any of the parameters are positive. Although the low predictive values that are associated with the considered parameters $(<1$ for bacteria, any positive nitrite test, presence of few or less esterase, and $<5$ WBCs) have been previously reported among outpatients [12, 17-18]. Our study also demonstrated that the criteria are ineffective in preventing unnecessary urine cultures among inpatients as well.

In the present study, we demonstrated that the UA reflex criteria are not effective in predicting positive urine cultures. The observed low predictive values are associated with the laboratory current UA reflex policy. Although the low predictive values that are associated with the considered parameters; ( presence for bacteria, any positive nitrite test, the presence of trace or few esterase, and the presence of WBCs) have been previously reported among out patients, this present study also demonstrated that the same criteria are ineffective in preventing unnecessary antibiotics therapy among inpatients as well $[3,16-17]$.

The study assessments pointed out the high rate of misuse of antibiotics when using suboptimal laboratory practices [18-19]. In particular, the data analysis indicated that a total of $45.1 \%$ of antibiotics were unnecessarily administrated, thus compromising patient safety and adding the risk of developing antibiotic resistance pathogens [20-24].

In conclusion, the current Laboratory UA reflex criteria is ineffective in predicting positive urine culture, thus potentially leading to the inappropriate use of antibiotics. Additionally, the assessment of the historical current UA reflex criteria confirmed the need for the refinement of the laboratory UA reflex practice to improve the quality of laboratory results and to provide accurate and reliable results to ensure adequate and optimal patient treatments.

\section{Conflict of Interest}

None declared

\section{Funding}

No funding

\section{References}

1. Altorf-van der Kuil W, Schoffelen AF, de Greeff SC, et al. National laboratory-based surveillance system for antimicrobial resistance: a successful tool to support the control of antimicrobial resistance in the Netherlands. Euro Surveill Bull Eur Sur Mal Transm Eur Commun Dis Bull 2017; 22.

2. Li B, Webster TJ. Bacteria antibiotic resistance: New challenges and opportunities for implant-associated orthopedic infections. J Orthop Res Off Publ Orthop Res Soc 2018; 36:22-32.

3. Agarwal R. Quality-Improvement Measures as Effective Ways of Preventing Laboratory Errors. Lab Med 2014; 45:e80-e88.

4. Centres for Disease Control and Prevention (US). Antibiotic resistance threats in the United States, 2013. 2013. Available at: https://www.cdc.gov/drugresistance/pdf/ar-threats-2013-508.pdf. 
5. Humphries RM, Dien Bard J. Point-Counterpoint: Reflex Cultures Reduce Laboratory Workload and Improve Antimicrobial Stewardship in Patients Suspected of Having Urinary Tract Infections. J Clin Microbiol 2016; 54:254-258.

6. Fok C, Fitzgerald MP, Turk T, et al. Reflex testing of male urine specimens misses few positive cultures may reduce unnecessary testing of normal specimens. Urology 2010; 75:74-76.

7. Adedeji WA. The Treasure Called Antibiotics. Ann lb Postgrad Med 2016; 14:56-57.

8. Adrizain R, Setiabudi D, Chairulfatah A. The inappropriate use of antibiotics in hospitalized dengue virus-infected children with presumed concurrent bacterial infection in teaching and private hospitals in Bandung, Indonesia. PLoS Negl Trop Dis 2019; 13:e0007438.

9. Centers for Disease Control and Prevention. Outpatient Antibiotic Prescriptions - United States, 2017. 2014. Available at: https://www.cdc.gov/antibiotic-use/community/pdfs/annualreportsummary 2014.

10. Aitken SL, Dilworth TJ, Heil EL, et al. Agricultural Applications for Antimicrobials. A Danger to Human Health: An Official Position Statement of the Society of Infectious Diseases Pharmacists. Pharmacotherapy 2016; 36:422-432.

11. Bergfeldt V. Microbes that never sleep: A multidisciplinary study of the antibiotic resistance management in Sweden.; 2016. Available at: http://urn.kb.se/resolve?urn=urn:nbn:se:sh:d iva-30623. Accessed May 28, 2020.

12. Alumran A, Hou $X-Y$, Hurst C. Validity and reliability of instruments designed to measure factors influencing the overuse of antibiotics. J Infect Public Health 2012; 5:221-232.

13. Ashiru-Oredope D, Hopkins S. Antimicrobial resistance: moving from professional engagement to public action. J Antimicrob Chemother 2015; 70:2927-2930.

14. Chang $\mathrm{Y}-\mathrm{Y}$, Chen $\mathrm{H}-\mathrm{P}$, Lin $\mathrm{C}-\mathrm{W}$, et al. Implementation and outcomes of an antimicrobial stewardship program: Effectiveness of education. J Chin Med Assoc JCMA 2017; 80:353-359.

15. Schroeder AR, Chang PW, Shen MW, et al. Diagnostic accuracy of the urinalysis for urinary tract infection in infants $<3$ months of age. Pediatrics 2015; 135:965-971.

16. Rabinovitch A, Clinical and Laboratory Standards Institute. Urinalysis: approved guideline. Wayne, Pa.: Clinical and Laboratory Standards Institute; 2009.
17. Richards KA, Cesario S, Best SL, et al. Reflex urine culture testing in an ambulatory urology clinic: Implications for antibiotic stewardship in urology. Int J Urol Off J Jpn Urol Assoc 2019; 26:69-74.

18. Jaeger C, Waymack J, Sullivan $P$, et al. Refining reflex urine culture testing in the ED. Am J Emerg Med 2019; 37:1380-1382.

19. Wold K, Brock J, Percival K, et al. Assessment of Reflex Urine Culture Criteria Changes and its Impact on Treatment of Asymptomatic Bacteriuria. Open Forum Infect Dis 2017; 4:S346-S346.

20. Yarbrough ML. Impact of Reflex Algorithms on Urine Culture Utilization. Clin Microbiol Newsl 2018; 40:19-24.

21. Barlam TF, Cosgrove SE, Abbo LM, et al. Implementing an Antibiotic Stewardship Program: Guidelines by the Infectious Diseases Society of America and the Society for Healthcare Epidemiology of America. Clin Infect Dis 2016; 62:e51-e77.

22. Islam J, Ashiru-Oredope D, Budd E, et al. A national quality incentive scheme to reduce antibiotic overuse in hospitals: evaluation of perceptions and impact. J Antimicrob Chemother 2018; 73:1708-1713.

23. Flores-Mireles AL, Walker JN, Caparon M, et al. Urinary tract infections: epidemiology, mechanisms of infection and treatment options. Nat Rev Microbiol 2015; 13:269-284.

24. Schmiemann G, Kniehl E, Gebhardt K, et al. The diagnosis of urinary tract infection: a systematic review. Dtsch Arzteblatt Int 2010; 107:361-367.

Publish in The International Arabic Journal of Antimicrobial Agents

The Journal is an open access peer-reviewed journal that publishes scientific papers about all aspects of antimicrobials. The journal will publish original research articles, reviews, brief reports and case reports dealing with basic and clinical antibacterial agents, antiviral, antiprotozoals, antituberculuous, antifungal and antihelminthes agents. All manuscripts must be prepared in English, and are subject to a rigorous and fair peer-review process. Accepted papers will immediately appear online. The journal aims to advance the knowledge, attitude and the research of chemotherapy in the Arabic world in cooperation with international, national scientific and public societies as well as research centers with similar aims and objectives. 